\title{
Spin Noise Spectroscopy in GaAs
}

\author{
M. Oestreich, M. Römer, R. J. Haug, and D. Hägele \\ Institut für Festkörperphysik, Universität Hannover, Appelstraße 2, D-30167 Hannover, Germany
}

(Received 18 May 2005; published 17 November 2005)

\begin{abstract}
We observe the noise spectrum of electron spins in bulk GaAs by Faraday-rotation noise spectroscopy. The experimental technique enables the undisturbed measurement of the electron-spin dynamics in semiconductors. We measure exemplarily the electron-spin relaxation time and the electron Landé $g$ factor in $n$-doped GaAs at low temperatures and find good agreement of the measured noise spectrum with a theory based on Poisson distribution probability.
\end{abstract}

DOI: 10.1103/PhysRevLett.95.216603

The inexorable decrease of structure size in semiconductor devices inevitably leads from today's quasiclassical devices to quantum mechanical devices. These quantum mechanical devices might rely not only on the charge of electrons, i.e., on the spatial part of the electron wave function, but also on the much more robust spin part of the wave function. The robustness of the electron spin motivates the current extensive research on the spin dynamics in semiconductors and might lead to spintronic devices with superior functionality and to the enchanting goal of spin quantum information processing [1-3].

One important signature of the spin dynamics in semiconductors results from the thermal fluctuations of electron-spin occupation in the conduction band which fluctuates on the time scale of the spin lifetime and gives rise to spin noise. This kind of spin noise has been observed recently in rubidium gas atoms [4] and theoretically exploited for spin currents through single quantum dots [5]. The experimental observation of spin noise in semiconductors is, however, a major challenge since the photon shot noise in optical experiments and the Coulomb shot noise in electrical experiments is usually orders of magnitude larger than the spin noise. On the other hand, measurement of the spin noise in semiconductors has the power to become an important experimental tool since the spin noise spectrum yields not only information about the electron Landé $\mathrm{g}$ factor but also promises to give insight into electron-electron spin correlations, spin phase transitions, and spin fluctuations in low-dimensional semiconductor structures. Spin noise spectroscopy is in certain circumstances orders of magnitudes more sensitive than electronspin resonance and according to our calculations even sensitive enough to detect the spin dynamics in a single semiconductor quantum dot. Additionally, spin noise spectroscopy has the advantage of detecting optically the spin dynamics in semiconductors without optical excitation of electrons and holes and can therefore be used, for example, to study the spin dynamics in Bose-Einstein condensates.

This Letter presents an experimental and theoretical description of spin noise spectroscopy in semiconductors. The experimental setup is schematically depicted in Fig. 1.
PACS numbers: 72.25.Rb, 72.70.+m, 78.47.+p, 78.55.Cr

The optical measurements are performed on a $370 \mu \mathrm{m}$ thick GaAs wafer with a silicon $n$ doping of $1.8 \times$ $10^{16} \mathrm{~cm}^{-3}$ by Faraday-rotation spectroscopy. The light of a cw laser diode is sent through a spatial filter, linearly polarized by a Glan Thompson polarizer, and focused on the GaAs sample which is mounted in Voigt geometry in a superconducting split-coil magnet with variable temperature insert. The laser wavelength is tuned $10 \mathrm{~nm}$ below the GaAs band gap to $826 \mathrm{~nm}$ to minimize absorption and maximize the Faraday-rotation signal. The focus diameter of the laser is about $65 \mu \mathrm{m}$. The linearly polarized light passing through the sample is split by a polarizing beam splitter into two components, linearly polarized $\pm 45^{\circ}$ with respect to the initial polarization. The two components are focused on a pair of photo diodes of a NewFocus $650 \mathrm{MHz}$ balanced photo receiver. The time varying difference of the two (equally strong if temporally averaged) components is converted with a gain of $350 \mathrm{~V} / \mathrm{W}$ into voltage and measured by an HP spectrum analyzer. The laser intensity before the beam splitter is $1.9 \mathrm{~mW}$ resulting in a white photon shot noise of $10 \mathrm{nV} / \sqrt{\mathrm{Hz}}$. The electrical noise of

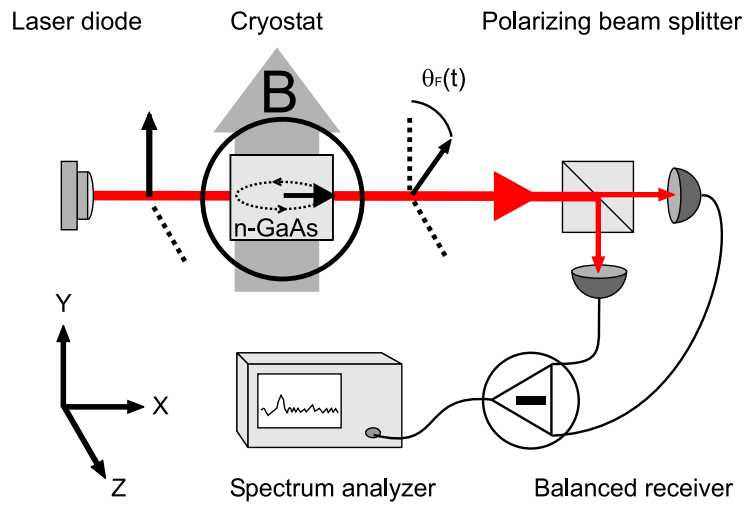

FIG. 1 (color online). Schematic of the experiment. Thermodynamic fluctuations of the electron spins in $n$-doped GaAs precess around an external magnetic field. The precessing spin fluctuations cause oscillations of the Faraday-rotation signal $\Theta_{F}$ whose power spectrum is detected by a balanced receiver and a spectrum analyzer. 
the combination of balanced receiver and spectrum analyzer is $13 \mathrm{nV} / \sqrt{\mathrm{Hz}}$ [6]. We can distinguish between the large external noise sources and the small spin noise by applying a weak magnetic field to the sample perpendicular to the direction of the laser light propagation. The spin noise strongly depends on the magnetic field since any statistical imbalance of the electron-spin ensemble precesses around the magnetic field resulting in a spin noise peak at the precession frequency. The width of the spin noise peak is proportional to the spin relaxation rate, which includes diffusion of the thermal spin fluctuations out of the laser focus. The white photon and electron noise powers do not depend on the magnetic field and therefore can be easily subtracted.

Figure 2 depicts the measured spin noise spectra for three magnetic fields. The power spectrum at each magnetic field is averaged over 62 measurements each taking $10 \mathrm{~min}$. and subtracted by 62 interleaving $10 \mathrm{~min}$. measurements at zero magnetic field to subtract the photon and electrical noise. We alternate between finite and zero magnetic field to eliminate any influence of thermal drift in the electronics. The spin noise maxima in Fig. 2 shift linearly with $B$ since the spin precession frequency and therefore the spin noise frequency is directly proportional to $B$. The width of the spin noise spectra is about $7 \mathrm{MHz}$ yielding a spin relaxation time of about $45 \mathrm{~ns}$. This spin relaxation

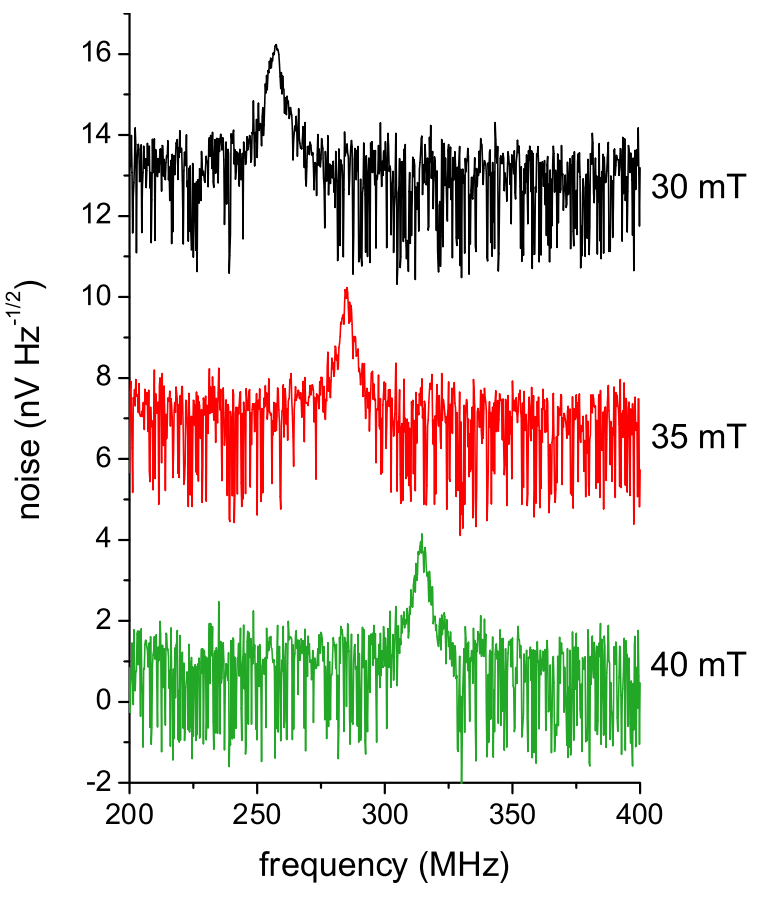

FIG. 2 (color online). Spectrum of the spin noise for different applied magnetic fields. The white photon shot noise and electrical noise power are subtracted. The spectra are vertically shifted for clarity. The sample temperature is $10 \mathrm{~K}$. Frequency and width of the spin noise spectrum are a direct measure of the electron Landé $\mathrm{g}$ factor and the electron-spin relaxation time, respectively. time is consistent with earlier measurements by Dzhioev et al. who measure $50 \mathrm{~ns}$ for localized donor bound electrons in GaAs with an $n$ doping of $1.5 \times 10^{16} \mathrm{~cm}^{-3}$ [7].

Figure 3 depicts the maxima of the spin noise peaks versus $B$. The spin precession frequency is equal to $g_{e} \mu_{B} B / h$, where $g_{e}$ is the electron Landé $\mathrm{g}$ factor, $\mu_{B}$ is Bohr's magneton, and $h$ is Planck's constant. The slope of the measured noise frequency $f$ versus $B$ yields $g_{e}=$ $-0.41 \pm 0.01$ which is in excellent agreement with optically oriented and detected electron-spin resonance in lightly doped $n$ GaAs [8], confirming that the measured spin noise is, in fact, correlated with donor bound electrons. The width of the noise spectra is a measure of the spin relaxation time (right inset in Fig. 3), which is within the measurement error independent of $B$, i.e., the spin relaxation measurement is not obscured by inhomogeneous broadening of $g_{e}$.

In the following, we want to theoretically estimate the amplitude of the spin noise and compare the results with our measurements. We make the simplifying assumption that the statistical fluctuation of the electron-spin polarization along the light propagation is proportional to $\sqrt{N}$, where $N$ is the number of donor bound electrons in the volume $V$ of the laser. In our experiment, this is a good starting point since the electrons are localized at the donors and the Poisson prerequisite of independent events is, therefore, if we neglect donor-donor interactions, in first approximation fulfilled. The experiment supports the assumption since we do not observe a temperature dependence of the width and the height of the noise peak when we increase the temperature from $5 \mathrm{~K}$ to $10 \mathrm{~K}$, i.e., the area under the noise peak stays constant. The localization of the electrons has additionally been verified by temperature dependent transport measurements.

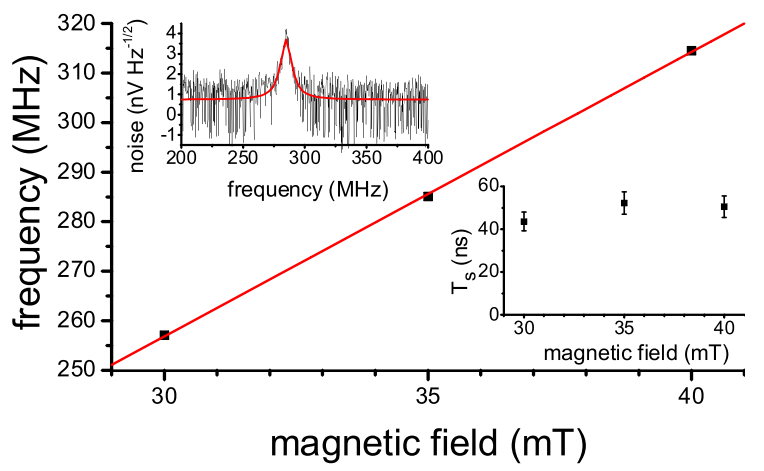

FIG. 3 (color online). Frequency of the maximum of the spin noise (filled squares) and spin relaxation time $T_{s}$ determined from the full-width at half-maximum (right inset) versus magnetic field $B$. The slope of the linear fit to the frequency (solid line) yields an electron Landé $\mathrm{g}$ factor of $-0.41 \pm 0.01$. The fit does not extrapolate to $f \rightarrow 0$ for $B \rightarrow 0$ due to a known constant remanent field of the superconducting magnet, which was not subtracted in this figure. The upper, left inset depicts the Lorentzian fit to the noise spectra at $35 \mathrm{mT}$. 
To estimate the influence of the statistical spin fluctuations on the refractive index we presume that the donor electrons change mainly the spin-dependent free $1 \mathrm{~s}$-exciton transition due to phase-space filling [9] and that the exciton transition is totally bleached when the electron Fermi energy is equal to the exciton binding energy $E_{b}=$ $5 \mathrm{meV}$. We neglect higher excitonic transitions since the exciton transition strength decreases with one over the third power of quantum number. We calculate the change of absorption $\Delta \alpha$ due to the statistical spin fluctuations by extending the so-called Elliott formula [10] to

$$
\begin{aligned}
\Delta \alpha(\hbar \omega)= & 0.5 \frac{e^{2} \hbar}{n_{r} \epsilon_{0} c m_{0} \hbar \omega} \frac{\left|p_{c v}\right|^{2}}{m_{0}} \frac{\delta\left(\hbar \omega-E_{b}\right)}{a_{e x}^{3}} \\
& \times \sqrt{N} /\left(V \int_{0}^{E_{b}} f(E) \operatorname{DOS}(E) d E\right)
\end{aligned}
$$

where $e$ is the electron charge, $n_{r}=3.665$ the refractive index, $\left|p_{\mathrm{cv}}\right|^{2}=2.1 \times 10^{-48} \mathrm{~kg}^{2} \mathrm{~m}^{2} / \mathrm{s}^{2}$ the average of the squared matrix element for transitions between Bloch states, $a_{\mathrm{ex}}=12 \mathrm{~nm}$ the exciton Bohr radius, $f(E)$ the Fermi-Dirac distribution, and $\operatorname{DOS}(E)$ the three dimensional density of states of the conduction band. The factor of 0.5 results from the optical selection rules in bulk GaAs which yield 50\% spin polarization for heavy hole and light hole and a given circular polarization. This model gives an upper limit for $\Delta \alpha$ since we are close to the metal insulator transition where excitonic effects are partially quenched and since nonexcitonic transitions are considerably weaker. Applying Kramers-Kronig relation to $\Delta \alpha$ yields the average change in refractive index for right- $\left(\sigma^{+}\right)$and left-circularly $\left(\sigma^{-}\right)$polarized light and thereby the average Faraday-rotation angle. The change of refractive index $\Delta n$ at $826 \mathrm{~nm}$ due to the thermal spin fluctuations becomes in our sample $\Delta n \approx 2.5 \times 10^{-8}$ and the resulting Faradayrotation angle $\Theta_{\mathrm{F}} \approx 7 \times 10^{-5} \mathrm{rad}$. The calculated maximum of the noise peak for a spin relaxation time of $45 \mathrm{~ns}$ is $9.5 \mathrm{nV} / \sqrt{\mathrm{Hz}}$. This calculated noise is for the simplicity of the calculation and the uncertainties in the experimental parameters in very good agreement with the measured $4 \mathrm{nV} / \sqrt{\mathrm{Hz}}$.

All measurements have been carried out at low temperatures where electrons are localized and the noise signal is temperature independent. At higher temperatures the donor bound electrons become delocalized and the distribution of the electrons in the conduction band follows a MaxwellBoltzmann distribution. The average size of fluctuations $\varsigma_{N^{+}-N^{-}}^{2}$ of the absolute spin orientation is expected to be for the Maxwell-Boltzmann distribution (as in the localized case) temperature independent and equal to $N$. Nevertheless, the Faraday-rotation signal should become temperature dependent since $\Delta \alpha$ broadens and moves in energy with increasing temperature. The independence of $\varsigma^{2}$ on temperature will change to a strong temperature dependence in samples with higher electron densities where the donor electrons are not localized anymore and the electrons may be described as free electrons in the conduction band with a Fermi-Dirac distribution $f(E)$ with

$$
\varsigma_{N^{+}-N^{-}}^{2}=V \int_{E_{\text {gap }}}^{\infty} \operatorname{DOS}(E) f(E)[1-f(E)] d E
$$

For Fermi-Dirac distributions,

$$
\lim _{T \rightarrow 0} \varsigma_{N^{+}-N^{-}}=0 \text { and } \quad \lim _{T \rightarrow \infty} \varsigma_{N^{+}-N^{-}}=\sqrt{N},
$$

with a most pronounced temperature dependence between $T=0$ and $k_{\mathrm{B}} T \leq E_{\mathrm{F}}$, where $k_{\mathrm{B}}$ is Boltzmann's constant and $E_{\mathrm{F}}$ is the Fermi energy.

We expect spin noise spectroscopy to have a wide range of applications in semiconductors. As an example we show in the following that spin noise is in principle capable to measure intrinsic electron-spin relaxation times at low temperature with less uncertainties than Faraday-rotation after optical excitation of spin or Hanle measurements. The intrinsic electron-spin relaxation time recently gained new interest, since experiments and calculations by Beck et al. at $4 \mathrm{~K}$ adumbrate that the electron-spin relaxation times in GaAs with an $n$ doping in the range between $10^{15}$ and $10^{16} \mathrm{~cm}^{-3}$ might not be limited by the anisotropic exchange interaction [11] but by the Dyakonov-Perel (DP) mechanism [12]. The DP mechanism vanishes for electrons with wave vectors $|\vec{k}| \rightarrow 0$. Therefore, low electron temperatures and the absence of additional spin relaxation mechanisms are required for long spin relaxation times. These requirements are extremely difficult to achieve in Faraday-rotation after optical excitation and Hanle measurements for two reasons. Firstly, the carrier temperatures are intrinsically higher than the sample temperature and secondly, the optically injected holes cause additional spin relaxation due to the well known Bir-Aronov-Pikus (BAP) mechanism [13]. The temperature of the optically injected carriers are at low sample temperatures intrinsically higher than the sample temperature since even resonant optical excitation (excitation of the $n$-doped semiconductor at the Fermi edge) yields hot holes with high $k$ values and carrier cooling at low temperatures is extremely inefficient due to the inefficient coupling of carriers with acoustic phonons [14]. Secondly, spin relaxation due to holes by the BAP mechanism is difficult to rule out since carrier recombination times become extremely long at low carrier concentration. The importance of BAP even at lowest pump intensities has been nicely demonstrated by several groups (see, e.g., Ref. [7], inset of Fig. 2). Spin noise measurements, on the other hand, do not necessarily excite carriers and thereby circumvent the above problems including the sometimes displeasing dynamical nuclear spin polarization. Since the amplitude of the spin noise signal is proportional to the spin relaxation time, spin noise spectroscopy particularly qualifies for systems with long spin relaxation times. 


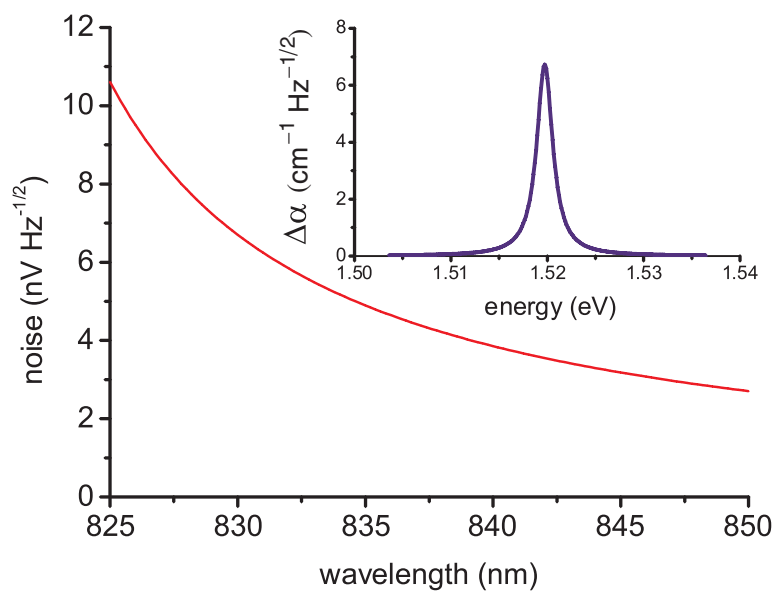

FIG. 4 (color online). Calculated maximum of the electronspin noise signal in dependence on detection wavelength for our experimental parameters. The same calculations for $p$-doped yields a noise power which is 5 orders of magnitude smaller due to the much faster spin relaxation of free holes. The inset depicts the change of absorption due to the thermal fluctuations of the electron spins in dependence on energy [Eq. (1)].

To evidence that spin noise measurements are sufficiently sensitive even below the band tail absorption, we have calculated the Faraday-rotation noise signal in dependence on wavelength (see Fig. 4). The noise power decreases by 1 order of magnitude when we increase the excitation wavelength from 826 to $840 \mathrm{~nm}$ and by 2 orders of magnitude when increasing to $900 \mathrm{~nm}$. At $826 \mathrm{~nm}$ about $90 \%$ of the light is absorbed in our $370 \mu \mathrm{m}$ thick GaAs sample yielding an excitation density of $5 \times 10^{14} \mathrm{~cm}^{-3}$ assuming a radiative carrier life time of $10 \mathrm{~ns}$. At $840 \mathrm{~nm}$ or even $900 \mathrm{~nm}$ the absorption is nearly negligible. We have also calculated the Faraday-rotation noise power for nonlocalized electrons, e.g., in modulation doped GaAs, and we find at the same electron temperature a comparable noise power and a similar decrease of 2 orders of magnitude at $900 \mathrm{~nm}$. We have to compare these decreases in noise power with the signal-to-noise ratio in Fig. 2. Keeping in mind that our present experiment is limited by digitalization errors of the spectrum analyzer and that these errors can be reduced by use of an additional analog amplifier, we are confident that even 2 orders of magnitude lower doping concentrations should be measurable since the noise power decreases only with the square root of the doping concentration. Further calculations surprisingly re- veal that even single quantum dot noise spectroscopy should be relatively easily feasible due to the very narrow absorption line width of the quantum dot.

In conclusion, we have measured the spin dynamics in $n$-doped bulk GaAs by spin noise spectroscopy. The sensitive technique allows the disturbance free measurement of the spin dynamics in semiconductors with high accuracy. The measurements show in combination with calculations that spin noise spectroscopy in semiconductors is a powerful tool, circumvents common problems of carrier heating and injection of interfering holes, and promises new insight into spin relaxation, magnetization dynamics, and electron-spin correlations.

This work has been supported by the Deutsche Forschungsgemeinschaft, the Alexander-von-Humboldt foundation, and the German Federal Ministry of Education and Research (BMBF) by the funding program NanoQUIT. We thank R. Winkler for helpful comments. M.O. is indebted to Axel Lorke and Pierre M. Petroff for stimulating discussions and enjoyable hospitality at the UCSB.

[1] S. A. Wolf et al., Science 294, 1488 (2001).

[2] J. M. Elzerman et al., Nature (London) 430, 431 (2004).

[3] M.I. Dyakonov, Future Trends in Microelectronics: The Nano Millenium, edited by $\mathrm{S}$. Luryi, J. $\mathrm{Xu}$, and $\mathrm{A}$. Zaslavsky, (Wiley, New York, 2002), pp. 307-318.

[4] S. A. Crooker, D. G. Rickel, A. V. Balatsky, and D. L. Smith, Nature (London) 431, 49 (2004).

[5] B. Dong, H.L. Cui, and X.L. Lei, Phys. Rev. Lett. 94, 066601 (2005).

[6] The spectrum analyzer exhibits a strong additional periodic structure on top of the power spectrum which is independent of the input signal and probably arises from digitalization errors. This additional structure is about 1 order of magnitude stronger than the electrical noise but can be also fairly reliably subtracted.

[7] R. I. Dzhioev et al., Phys. Rev. B 66, 245204 (2002).

[8] J. S. Colton et al., Phys. Rev. B 67, 165315 (2003).

[9] D. Huang, Jen-Inn Chyi, and H. Morkoc, Phys. Rev. B 42, 5147 (1990).

[10] R. J. Elliott, Phys. Rev. 108, 1384 (1957).

[11] K. V. Kavokin, Phys. Rev. B 64, 075305 (2001).

[12] M. Beck, C. Metzner, S. Malzer, and G. H. Döhler, condmat/0504668.

[13] G. E. Pikus, A. G. Aronov, and G. E. Pikus, Zh. Eksp. Teor. Fiz. 69, 1382 (1975) [Sov. Phys. JETP 42, 705 (1976)].

[14] D. von der Linde and R. Lambrich, Phys. Rev. Lett. 42, 1090 (1979). 